\title{
Caracterización de la producción científica de Ecuador en el periodo 2007-2017 en Scopus
}

\author{
Liggia Moreira-Mieles* \\ Juan Carlos Morales-Intriago* \\ Sofía Crespo-Gascón**

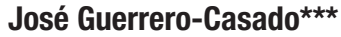

Artículo recibido:

4 de marzo de 2019

Artículo aceptado:

25 de junio de 2019

Artículo de investigación

\section{Resumen}

Ecuador ha aumentado vertiginosamente su producción científica en los últimos años, actualmente es el sexto país de Latinoamérica y el Caribe con mayor número de artículos publicados en Scopus. El objetivo de este trabajo fue estudiar la evolución de la producción científica de Ecuador en Scopus durante el periodo 2007-2017, obteniendo para cada año el número de citas recibidas, el porcentaje de artículos por cuartil, el porcentaje de artículos escritos en inglés, español u otros idiomas, y el porcentaje de documentos publicados como artículos, revisiones y memorias de congresos, entre

\footnotetext{
* Facultad de Ciencias Humanísticas y Sociales, Universidad Técnica de Manabí. Ecuador Imoreira0888@utm.edu.ec_jcmorales@utm.edu.ec ** Instituto de Idiomas, Universidad Técnica de Manabí. Ecuador

screspo@utm.edu.ec

*** Facultad de Ciencias Veterinarias, Universidad Técnica de Manabí. Ecuador

guerrero.casado@gmail.com

INVESTIGACIÓN BIBLIOTECOLÓGICA, vol. 34, núm. 82, enero/marzo, 2020, México, ISSN: 2448-8321 pp. 141-157
} 
otros. Los resultados muestran un aumento en la proporción de artículos en Q4 y un descenso en Q1, un aumento en la proporción de artículos escritos en español, y un aumento del porcentaje de documentos publicados como memorias de congreso, mientras que no hubo evidencia de un aumento en el número de citas recibidas. Estos resultados sugieren que el aumento de la producción científica en Ecuador no ha dado como resultado un aumento de la calidad, medida como usabilidad (citas), visibilidad (idioma) e impacto (cuartiles), lo que podría ser resultado de la incipiente ciencia que se está generando en el país.

Palabras clave: Análisis Bibliométrico; Citas; Calidad Científica; Producción Científica

\section{Characterization of the scientific production in Ecua-} dor for the period 2007-2017 in Scopus

Liggia Moreira-Mieles, Juan Carlos Morales-Intriago, Sofía Crespo-Gascón and José Guerrero-Casado

\section{Abstract}

Ecuador has dramatically increased its scientific production in the last years, being the sixth country of Latin America and The Caribbean with greater number of articles indexed in Scopus. The objective of this work was to study the evolution of scientific production in Ecuador in Scopus for the period 2007-2017, obtaining for each year the number of citations, the percentage of articles in the different quartiles, the percentage of articles written in English, Spanish or other languages, and the percentage of documents published as articles, reviews or conferences and proceedings among others. The results showed an increase in the proportion of articles in Q4 and a decrease in Q1; an increase in the proportion of articles written in Spanish; an increase in the percentage of articles published as conferences and proceedings; and there is no evidence on the amount of the citations. These results suggest that the increase in the scientific production in Ecuador does not entail an increase in the quality measured as usability (citations), visibility (language), and impact (quartile), which could be a result of the incipient science that is being generated in the country.

Keywords: Bibliometric Analysis; Citations; Scientific Production; Scientific Quality 


\section{INTRODUCCIÓN}

T a región latinoamericana comprende un total de 46 países, no obstante, Lu producción científica representa un bajo porcentaje en relación a la producción mundial. Entre los factores que explican esta baja contribución podemos resaltar la ínfima inversión en investigación y desarrollo (I+D), escasa inversión privada en actividades científico-tecnológicas, un bajo número de profesionales dedicados a la investigación y el desarrollo tecnológico, o el mayor costo de materiales y equipamientos científicos de ciertos países que conforman esta región (Bonilla, Merigó y Torres-Abad, 2015; Chinchilla-Rodríguez et al., 2015; Guerrero-Casado, 2017). La Comisión Económica para América Latina y el Caribe (CEPAL), sobre la base de información de la Organización de las Naciones Unidas para la Educación, la Ciencia y la Cultura (Unesco) y la Red de Indicadores de Ciencias y Tecnología Iberoamericana e Interamericana (RICYT), con respecto al PIB identifican cinco grupos de países según la intensidad de su inversión en $\mathrm{I}+\mathrm{D}$ : el primero corresponde a países desarrollados, con niveles superiores a $2 \%$; en el segundo grupo, entre $1 \%$ y $2 \%$, están España y Noruega, entre otros; en el tercer conjunto están Argentina, Costa Rica y México con una inversión de entre 0.5 \% y 1 \%; el cuarto grupo, con valor de entre $0.2 \%$ y $0.5 \%$, incluye países como Cuba, Chile, Ecuador, Uruguay y Colombia, y por último, con menos de $0.2 \%$, están países como Panamá y El Salvador (CEPAL, 2016: 21).

Sin embargo, un estudio realizado por Álvarez-Muñoz y Pérez-Montoro (2016) indica que si se realiza un análisis por regiones de aquellos documentos científicos indexados en Scopus por Scimago Journal \& Country Rank (SJR) entre el periodo 2010-2014, las cuatro zonas con un mayor incremento en producción científica son África, Oriente Medio, Asia y Latinoamérica, con porcentajes de $36.5 \%$, 28.81\%, $24.3 \%$ y $17.69 \%$ respectivamente, superando a Europa y Norteamérica, regiones que son mayores productoras de ciencia. Entre los países que más han aumentado la producción científica de Latinoamérica destaca Ecuador, un país con poca tradición en este ámbito pero cuya producción científica ha tenido un aumento vertiginoso a lo largo de los últimos años, con un incremento de $811.49 \%$ de documentos publicados (Tabla 1). De hecho, la tasa de crecimiento anual compuesta de Ecuador es la más alta de la región (Castillo y Powell, 2019), aunque hay que tener en cuenta que se parte de un bajo número de publicaciones comparado con la estabilización que se está alcanzado en otros países de la región. Según datos de Scimago, Ecuador se encontraba en el puesto 12 para 2007, posicionándose en la actualidad en el puesto 6 de la región (Tabla 1), sobrepasando a Perú, Cuba y Venezuela. Así, Ecuador ha pasado de contribuir 0.49 \% de la producción científica regional en 2007 a 2.27 \% en 2017 (Tabla 1). 


\begin{tabular}{|c|c|c|c|c|c|c|}
\hline Año & $\begin{array}{c}\text { Posición } \\
\text { LAyC }\end{array}$ & $\begin{array}{c}\mathrm{N}^{\circ} \text { de } \\
\text { artículos }\end{array}$ & $\begin{array}{c}\text { Contribución } \\
\text { artículos LAyC } \\
(\%)\end{array}$ & $\begin{array}{c}\text { Diferencia } \\
\text { crecimiento } \\
(\%)\end{array}$ & $\mathrm{N}^{\circ}$ de citas & $\begin{array}{c}\text { Contribución } \\
\text { citas LAyC } \\
(\%)\end{array}$ \\
\hline 2007 & 12 & 348 & 0.49 & 8.33 & 7795 & 0.61 \\
\hline 2008 & 12 & 416 & 0.51 & 19.54 & 14928 & 1.07 \\
\hline 2009 & 12 & 500 & 0.56 & 20.19 & 10710 & 0.79 \\
\hline 2010 & 12 & 457 & 0.48 & -8.6 & 11842 & 0.92 \\
\hline 2011 & 12 & 484 & 0.47 & 5.91 & 7763 & 0.63 \\
\hline 2012 & 12 & 648 & 0.58 & 33.88 & 9765 & 0.78 \\
\hline 2013 & 11 & 762 & 0.64 & 17.59 & 9278 & 0.94 \\
\hline 2014 & 10 & 1047 & 0.82 & 37.4 & 7765 & 0.94 \\
\hline 2015 & 9 & 1657 & 1.28 & 58.26 & 7863 & 1.26 \\
\hline 2016 & 6 & 2384 & 1.74 & 43.87 & 5392 & 1.56 \\
\hline 2017 & 6 & 3172 & 2.27 & 33.05 & 1563 & 2.3 \\
\hline Promedio & & 1079.55 & 0.89 & 24.49 & 8606 & 1.07 \\
\hline Desv Esta & & 935.687 & 0.608 & 19.068 & 3452.86 & 0.491 \\
\hline
\end{tabular}

Tabla 1. Posición de Ecuador con respecto a otros países de Latinoamérica y el Caribe de acuerdo al número de artículos publicados en Scopus según datos de Scimago Journal \& Country Rank. $n^{0}$ de artículos publicados por año, contribución (\%) a la producción científica de Latinoamérica y el Caribe (LAyC), diferencia porcentual (\%) de crecimiento interanual ((año $n$ - año n-1)/año $\left.n-1)^{*} 100\right), n^{0}$ de citas recibidas por año y contribución de las citas recibidas por artículos de Ecuador a las citas recibidas por todos los países de la región ( $\left.\left(n^{\circ} \text { citas Ecuador/ } \sum \text { citas Latinoamérica }\right)^{*} 100\right)$

Fuente: elaboración propia con datos extraídos de Scimago Journal \& Country Rank en el periodo 2007-2017

En Ecuador las universidades han experimentado una serie de cambios que han hecho que su papel y relación con la sociedad de cierta manera varíe, estos cambios han aportado a la construcción de un nuevo marco de políticas públicas y la concepción de un nuevo escenario en el cumplimiento de los objetivos de desarrollo (Toscanini Segale, Aguilar Guzmán y García Sánchez, 2016). Esto surge a raíz de la incorporación del Estado Social Democrático con la Constitución de 2008, la cual en su artículo 350 establece que

El sistema de educación superior tiene como finalidad la formación académica y profesional con visión científica y humanista; la investigación científica y tecnológica; la innovación, promoción, desarrollo y difusión de los saberes y las culturas; la construcción de soluciones para los problemas del país, en relación con los objetivos del régimen de desarrollo. 
Es por esta razón que el gobierno, por medio de las instituciones públicas de educación superior (IES), ha implementado en este sistema educativo políticas para el desarrollo de la ciencia en el país (Álvarez-Muñoz y Pérez-Montoro, 2016). Una de estas políticas es la creación del Consejo de Evaluación, Acreditación y Aseguramiento de la Calidad de la Educación Superior (CEAACES) como responsable de llevar a cabo los procesos diseñados para la evaluación del desempeño institucional de las universidades y escuelas politécnicas (CEAACES, 2015). El proyecto Prometeo fue también una de las políticas públicas que se implementó como iniciativa promovida por la Secretaría Nacional de Educación Superior, Ciencia y Tecnología (SENESCYT), con el objetivo de fortalecer zonas estratégicas del país con el desarrollo de las capacidades de investigación de las instituciones públicas y educativas, a través de la vinculación de investigadores extranjeros y ecuatorianos residentes en el exterior con reconocida trayectoria para desarrollar proyectos que aporten en áreas prioritarias del conocimiento, en conjunto con la institución de acogida (SENESCYT, 2015; Van Hoof, 2015). Otra política pública es la articulación del Sistema Nacional de Fomento al Talento Humano al sistema de Educación Superior mediante becas, créditos educativos, ayudas económicas, fondos de garantías y redes de becarios (SENESCYT, 2012), otorgándose en el periodo 2007-2015 18958 becas (SENESCYT, 2016). Además, en 2013, con una inversión inicial de 1100 millones de dólares, se crearon cuatro universidades denominadas emblemáticas como propuesta para pasar de una economía extractivista a una de recursos ilimitados basada en el conocimiento, creatividad e innovación, como la Ciudad del Conocimiento Yachay especializada en investigación científica, la Universidad Regional Amazónica (IKIAM) especializada en ciencias de la vida y ciencias de la Tierra, la Universidad Nacional de Educación para la formación de educadores y la Universidad de las Artes para la promoción del arte y la cultura (SENESCYT, 2013).

Sin embargo, son pocos los estudios que caracterizan la producción científica del Ecuador. Según trabajos previos, las políticas públicas que han estimulado la ciencia generada en las universidades (Álvarez-Muñoz y Pérez-Montoro, 2015) así como la producción en colaboración con países de mayor tradición en investigación (Castillo y Powell, 2018) han sido uno de los factores determinantes para aumentar la cantidad e impacto de los artículos publicados. En un reciente trabajo, Castillo y Powell (2019) demostraron que $80 \%$ de los artículos científicos publicados en Scopus por autores ecuatorianos fueron realizados en colaboración con investigadores de otros países, demostrando así los beneficios que el Ecuador recibe de la colaboración internacional. Por lo tanto, el objetivo del presente estudio es caracterizar la evolución de la producción científica del Ecuador en Scopus en el periodo 
2007-2017, considerando las variables citas, cuartiles, idioma y tipo de documento, con el objetivo de comprobar si el aumento en la cantidad se ve reflejado en un aumento del impacto (cuartil), visibilidad (idioma) y usabilidad (citas) como aspecto primordial para que la investigación contribuya a la comunidad científica y académica nacional e internacional.

\section{Metodología}

El objetivo de este trabajo es caracterizar la evolución de la producción científica del Ecuador mediante un análisis cuantitativo retrospectivo, considerando los artículos publicados en la base de datos Scopus (https://www.scopus. $\mathrm{com} /$ ), de donde se extraen aquellos artículos (fecha de búsqueda diciembre de 2018) que cumplan las condiciones en cuanto al periodo (2007-2017) y país de filiación (Ecuador); el resultado fueron 12237 artículos. Se eligió este periodo de tiempo para observar tendencias, además coincide con el momento en el cual más aumentó la producción científica del país. De cada trabajo se obtuvieron los datos siguientes: número de citas, idioma y tipo de documento. Finalmente, para cada año se obtuvo la siguiente información:

- Número medio de citas recibidas por los artículos escritos en cada año, dividiendo el número de citas por los años que el artículo lleva publicado.

- Porcentaje de artículos publicados en los distintos cuartiles (Q1, Q2, Q3 y Q4). Para cada artículo se comprobó en qué cuartil estaba la revista en el año en que se publicó con los datos procedentes del portal Scimago Journal \& Country Rank. En el caso de que la revista esté clasificada en varias categorías, se escogió el cuartil más alto.

- Porcentaje de documentos según el tipo: artículos, conferencias y memorias de congresos, revisiones, capítulos de libro y libros, cartas al editor y otros.

- Porcentaje de artículos escritos en los distintos idiomas, agrupándolos en tres grupos: inglés, español y otros.

\section{Resultados}

El número de artículos en Scopus aumentó significativamente puesto que en 2007 se contaban 348 artículos publicados y para 2017 se publicaron 3470 artículos. Ecuador no ha tenido un aumento de cita por años, entre 2007 y 
2013 se observa oscilación entre un año y otro, y a partir de 2014 en adelante hay un descenso paulatino. En 2013 el número medio de citas por documento fue 3.83; para el año 2017, 2.78 (Figura 1).

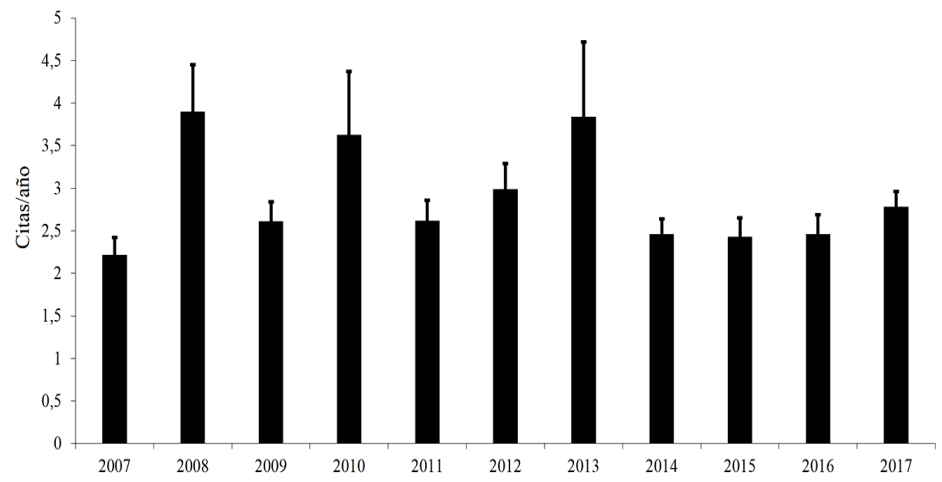

Figura 1. Número medio de citas recibidas por los artículos publicados en cada año durante el periodo 2007-2017

Fuente: elaboración propia, a partir de los datos obtenidos en Scopus

A lo largo de los 11 años el porcentaje más alto de los artículos publicados se ha presentado en el Q1 con 51.9 \% de media; sin embargo, desde 2012 hay un descenso moderado en el porcentaje de artículos del primer cuartil (Q1), pasando de $54.47 \%$ en 2012 a $41.3 \%$ en 2017, y por el contrario los artículos de Q4 pasan de $8.19 \%$ en 2014 a $16.7 \%$ en 2017 (Figura 2).

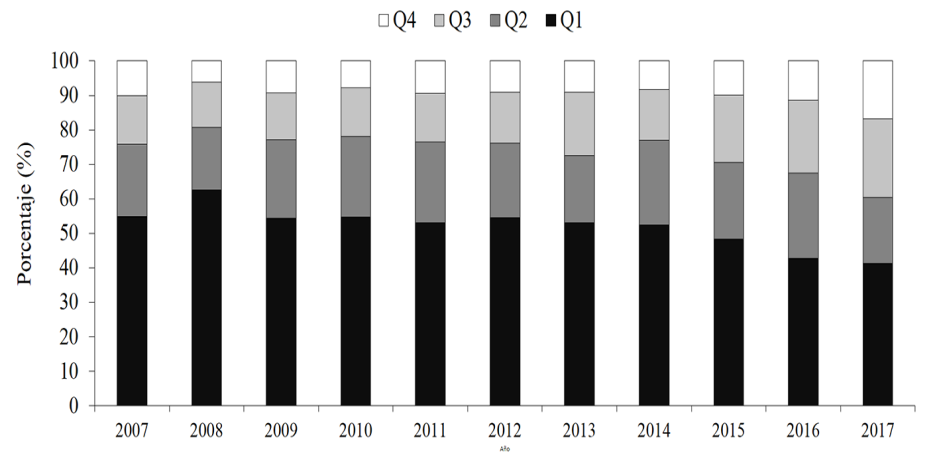

Figura 2. Porcentaje del cuartil en el que han sido publicado los artículos en cada año para el periodo 2007-2017

Fuente: elaboración propia, a partir de los datos de Scopus y Scimago Journal \& Country Rank 
En cuanto al idioma, en $200789 \%$ se escribían en inglés y $10 \%$ en idioma español, mientras que para $201780 \%$ se escribieron en inglés y $18 \%$ en español, evidenciando un aumento de la presencia de artículos escritos en español y por ende un descenso en el porcentaje de artículos escritos en inglés (Figura 3).

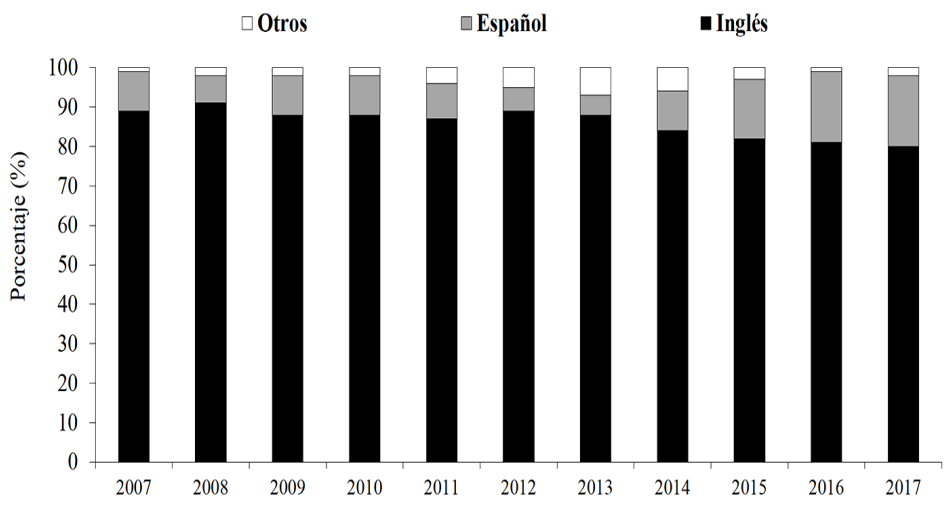

Figura 3. Idioma de los artículos publicados para cada año en el periodo 2007-2017 Fuente: elaboración propia, a partir de los datos obtenidos en Scopus

Por último, el principal tipo de documento fue el artículo científico, con una media de $74.8 \%$ durante los 11 años de estudio. Sin embargo, se observa un aumento en la proporción de documentos publicados como memorias de congresos (Conferences and Proceedings), pasando de $7.5 \%$ en 2007 a $26.6 \%$ en 2017 (Figura 4).

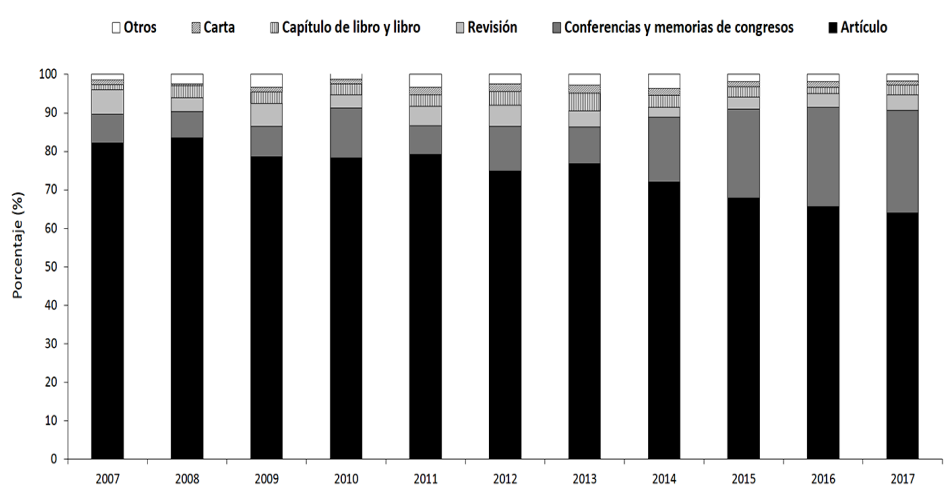

Figura 4. Porcentaje del tipo de documento para cada año en el periodo 2007-2017 Fuente: elaboración propia, a partir de los datos obtenidos en Scopus 


\section{Discusión}

La producción científica del Ecuador en el contexto latinoamericano ha tenido un aumento considerable, pasando de contribuir $0.49 \%$ en 2007 a $2.27 \%$ de la producción científica regional en 2017 (Tabla 1). Este aumento podría explicarse en parte por la presión que los investigadores con filiación ecuatoriana sienten por aumentar la producción científica de la institución para la que laboran, puesto que el CEAACES evaluaba de forma independiente la producción científica en Scimago o ISI Web of Knowledge, teniendo mayor peso que la producción en bases de datos regionales como Latindex, Scielo y Redalyc, entre otras (CEAACES, 2015). Además, las publicaciones son necesarias para acceder a los concursos de mérito y oposición para laborar como docente e investigador en las IES de Ecuador (CES, 2012) y acreditarse como investigador a nivel nacional (SENESCYT, 2018). Por ejemplo, para poder ser personal académico titular principal de las universidades y escuelas politécnicas es necesario tener publicadas al menos 12 obras de relevancia o artículos indexados (CES, 2012: artículo 32).

Sin embargo, la cantidad de artículos producidos no siempre conlleva una mayor calidad, además de no reflejar necesariamente el impacto real en la evolución de la ciencia, ni en el desarrollo humano y social (Fleitas Triana, Hernández Oquendo y Guerra Castillo, 2017; Tejedor-Estupiñán y Tejedor-Estupiñán, 2019). Así, por ejemplo, si hacemos un análisis de los datos de producción científica de los 12 países de Latinoamérica con mayor producción para el periodo 1996-2017 en el portal Scimago (SJR), los cuatro países con mayor producción son Brasil, México, Argentina y Chile, mientras que los países con mayor número de citas por documento son Puerto Rico, Costa Rica, Uruguay y Perú (Figura 5).

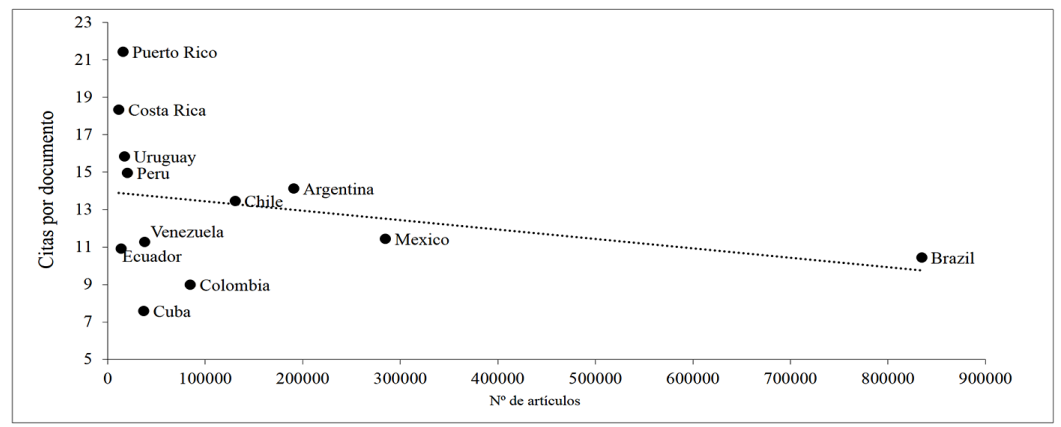

Figura 5. Relación entre el número de artículos producidos y el número de citas por documento de los 12 países de América Latina y el Caribe con mayor producción científica en Scopus para el periodo 1996-2017

Fuente: elaboración propia, a partir de los datos de Scimago Journal \& Country Rank 
Esto significa que los países más productores en volumen no son los que más citas reciben por documento, y por lo tanto, una mayor cantidad de publicaciones no implica una mayor calidad medida como el número de citas recibidas. En consecuencia, esta velocidad de crecimiento en la producción científica ecuatoriana podría estar afectando la calidad de su contenido en cuanto a usabilidad, visibilidad e impacto. Los resultados de este trabajo sugieren un descenso de la calidad de las publicaciones al encontrar un porcentaje menor del número de artículos en cuartiles altos, y no hay evidencia de un aumento en el número de citas que reciben los artículos. Además, se observó un mayor número de artículos escritos en español y de artículos publicados como memorias de congresos, lo cual podría afectar también la visibilidad de la ciencia ecuatoriana por ser documentos menos citados (ver más adelante). Resultados similares han sido reportados para Cuba durante el periodo 2003-2011, pues aunque aumentó la producción científica en Scopus hubo también un descenso del número de artículos en Q1, un descenso del número de citas por documento y un aumento de artículos escritos en español (Chinchilla-Rodríguez et al., 2015).

Aunque los datos muestran que un aumento en la cantidad no se refleja en la calidad, quizás este aumento en la producción sea un paso necesario para el desarrollo de la ciencia en Ecuador, previo a generar un sistema más maduro con mayor impacto (Loyola, Diniz-Filho y Bini, 2012). Idealmente, el sistema de producción científica en Ecuador debería ir encaminado a conseguir influencia, esto es, producir ciencia que suponga un avance científico y a la vez contribuya a resolver un problema en particular (Donaldson y Cooke, 2014), teniendo los científicos la responsabilidad de ayudar a la sociedad a conseguir un futuro mejor (Fischer, Ritchie y Hanspach, 2012). En este sentido es importante destacar que la Ley Orgánica de Educación Superior de Ecuador (LOES, 2010), en su artículo 107, establece que:

El principio de pertinencia consiste en que la educación superior responda a las expectativas y necesidades de la sociedad, a la planificación nacional, y al régimen de desarrollo, a la prospectiva de desarrollo científico, humanístico y tecnológico mundial, y a la diversidad cultural. Para ello, las instituciones de educación superior articularán su oferta docente, de investigación y actividades de vinculación con la sociedad, a la demanda académica, a las necesidades de desarrollo local, regional y nacional.

Este principio ha sido recogido en la Política de Evaluación Institucional de Universidades y Escuelas Politécnicas en el marco del Sistema de Aseguramiento de la Calidad de la Educación Superior (CACES, 2018), y es de 
esperar que en el nuevo modelo de evaluación de las IES, los artículos de los investigadores ecuatorianos estén vinculados tanto a la docencia como a la sociedad.

En cuanto al impacto, se ha observado una disminución de la proporción de artículos publicados en el cuartil 1 (Q1), y a su vez un aumento de los artículos en el cuartil 4 (Q4). Este hecho también podría estar ligado tanto a la necesidad por publicar como al "despertar" de la ciencia ecuatoriana, que al ser una ciencia con poca tradición tendrá más dificultades para poder publicar en revistas del alto impacto (Q1) en comparación con países de Europa o Estados Unidos, los cuales tienen una mayor inversión en investigación (Monge-Nájera, 2002). Por estos y otros motivos, los autores e instituciones de un país en vías de desarrollo como Ecuador no deberían ser evaluados bajo el mismo baremo que los países desarrollados con mayor inversión en $\mathrm{I}+\mathrm{D}$, esto es, por el factor de impacto de la revista donde el trabajo es publicado. Además, el factor de impacto no sólo ha sido criticado por investigadores de países en vías de desarrollo, sino también por investigadores de los países con mayor producción científica (Wouters et al., 2019). Aunque obviamente hay que incitar a los investigadores ecuatorianos a publicar en revistas bien posicionadas, el impacto de sus investigaciones no debería medirse únicamente por la posición de la revista en la que se publican sus trabajos. Como sugiere Monge-Nájera (2014), una solución podría ser crear un Índice Latinoamericano de Impacto más acorde con la realidad de la ciencia de la región, donde no hay que olvidar que una parte importante de la literatura científica se encuentra en revistas no indexadas en Scopus pero sí incluidas en bases de datos regionales como Latindex, Redalyc o Scielo (Miguel, 2011; Crespo-Gascón, Tortosa y Guerrero-Casado, 2019).

En cuanto al número de citas, no parece haber un incremento en el que reciben los documentos, existiendo oscilaciones interanuales (Figura 1). Hay que tener en cuenta que, aunque los datos están estandarizados por los años que llevan los artículos publicados, el patrón normal de citación hace que los artículos más recientes no tengan tiempo suficiente para ser citados en comparación con los artículos publicados con anterioridad. Otro aspecto a destacar es que el aumento de Ecuador a la contribución de los artículos publicados por países de Latinoamérica y el Caribe ha ido en paralelo con su contribución a las citas que los artículos de esta región reciben (Tabla 1), aunque hay que matizar que de 2007 a 2013 el porcentaje de contribución de las citas de los artículos de Ecuador era mayor que el porcentaje de contribución del número de documentos, siendo muy semejantes ambos porcentajes a partir de 2014. En otras palabras, entre 2007 y 2013 Ecuador contribuía a la región con más citas de lo que le correspondía según los artículos que publicaba, pero a partir de 2014 contribuye de manera muy similar a los documentos y citaciones de los artículos de la región. 
Así como la producción científica ha aumentado en el Ecuador, los documentos publicados como conferencias y memorias de congreso también han aumentado, siendo el segundo tipo de documento con mayor número de publicaciones tras el artículo. Esto se debe a que en ciertas áreas de la ciencia las conferencias y memorias de congresos son particularmente importantes, con la misma relevancia que un artículo publicado en revistas científicas (Castillo, 2018). Tal aumento ha sido reportado por Castillo (2018), quien sugiere que este hecho podría deberse a que el reglamento nacional considera como mérito relevante la publicación de artículos en memorias de congresos y por ser una tarea relativamente más fácil que la publicación de artículos científicos en revistas. Según los lineamientos para establecer el nivel de las publicaciones indexadas para el proceso de categorización y recategorización de investigadores (SENESCYT, 2018), las publicaciones en memorias de congresos se establecen en el nivel 2 si están incluidas en la base de datos Scopus, y en nivel 3 si no lo están, quedando al mismo nivel de las revistas indexadas en bases de datos regionales. Además, en el Reglamento de Carrera y Escalafón del Profesor e Investigador del Sistema de Educación Superior (CES, 2012), en su artículo 80 , para la determinación de la relevancia y pertinencia de las obras publicadas, considera los trabajos publicados en congresos y seminarios que cuenten con algunos criterios de calidad (ISBN, revisión por pares y comité científico), lo que indica que las comunicaciones en extenso en congreso computan para los concursos de mérito y oposición y para los procesos de promoción interna (escalafón). Sin embargo, según Castillo (2018), para el caso de Ecuador los artículos publicados en Scopus como conferencias reciben menos citas que los artículos convencionales en revistas científicas, lo cual ha sido también demostrado previamente por otros autores (González-Albo y Bordons, 2011; Zhang y Glänzel, 2012). Por lo tanto, este aumento en el número de publicaciones en memorias de congreso podría estar afectando la visibilidad y usabilidad de los artículos de autores ecuatorianos, por lo que sería recomendable que este tipo de publicación tenga menos peso en la normativa nacional. A modo comparativo, según datos obtenidos de Scopus, Perú y Colombia publicaron en $201715.4 \%$ y $14.5 \%$ de sus documentos en Scopus como memorias de congresos, frente a $26.6 \%$ de Ecuador.

Otro aspecto evidenciado en este trabajo es el importante aumento del número de artículos con filiación ecuatoriana escritos en español, lo cual podría estar disminuyendo la visibilidad de las publicaciones por el idioma, así como el número de citas que reciben. Se ha demostrado científicamente que los artículos que reciben más citas son aquellos que se encuentran escritos en inglés, por encima de aquellos que se escriben en español (Chinchilla-Rodríguez et al., 2015; Tahamtan, Safipour y Ahamdzadeh, 2016). Por 
ejemplo, en un reciente estudio, se demostró que en dos revistas españolas de Biblioteconomía y Documentación incluidas en el Journal Citation Reports (JCR) que publican en español, sólo una de cada 10 citas proceden de autores no iberoamericanos (Salvador Oliván, Marco-Cuenca y Arquero-Avilés, 2018). Además, es mucho más difícil publicar un artículo escrito en español en las revistas mejor posicionadas, por lo que el idioma también podría influir en el impacto. Este hecho también podría explicarse por la necesidad de los investigadores por tener artículos publicados para los procesos de acreditación como investigadores y acceso a concursos de méritos y oposición, lo que les podría llevar a escribir en su lengua natal, que resulta en un menor esfuerzo en relación con escribir en idioma inglés. Además, como muchos de los profesores son nóveles investigadores, puede que carezcan de la formación y experiencia necesaria para poder publicar en inglés. En definitiva, el aumento del número de artículos en español podría estar afectando a los tres indicadores (usabilidad, visibilidad e impacto), por lo tanto es recomendable que se establezcan programas formativos para fomentar la escritura de artículos en inglés.

Finalmente, es importante resaltar las limitaciones de este trabajo para comprender correctamente los resultados y sus implicaciones. En primer lugar, este trabajo muestra tendencias generales durante 11 años para el número de citas, idioma, cuartil y tipo de documento; sin embargo, los resultados pueden variar si el análisis de estas variables se realiza de forma independiente por área de conocimiento, ya que según el área en cuestión los resultados podrían ser diferentes a las tendencias generales que mostramos en este trabajo. En segundo lugar, existen muchas relaciones entre indicadores bibliométricos que podrían ayudar a identificar los factores que afectan al impacto de la ciencia de Ecuador, como por ejemplo la relación entre el cuartil y las citas recibidas con el idioma, el número de autores, la colaboración con autores de otros países, las fuentes de financiación o el país de afiliación del primer autor y el autor de correspondencia (Chinchilla-Rodríguez, Miguel y de Moya-Anegón, 2015; Chinchilla-Rodríguez et al., 2015; Castillo y Powell, 2019). A pesar de ello, consideramos que las tendencias generales aquí mostradas pueden servir de marco referencial para conocer la evolución de la producción científica ecuatoriana.

\section{Conclusiones}

A pesar del aumento de la producción científica en Ecuador en la última década, en este trabajo hemos demostrado que hay algunos indicadores bibliométricos relacionados con la usabilidad (no hay un incremento aparente 
del número de citas y hay un aumento de la proporción de documentos como memorias de congresos), impacto (menor proporción de artículos en Q1) y visibilidad (mayor proporción de artículos en español) que podrían estar repercutiendo negativamente. Parte de esta tendencia podría deberse como resultado de la incipiente ciencia que se está desarrollando en el país. Por lo tanto, la cantidad no debería ser el único indicador medible de la producción científica de las instituciones e investigadores ecuatorianos, y otros criterios como el número de citas, la contribución de la ciencia al sector productivo e industrial, la relación de los documentos con la docencia y las necesidades sociales, el establecimiento de redes de investigación nacional e internacional o el fomento de áreas de conocimiento con escasa trayectoria en Ecuador también deberían ser considerados, aunque siempre dentro del contexto en el que se desarrolla la ciencia en Latinoamérica.

Agradecimientos

Agradecemos al doctor Exio Chaparro por sus comentarios sobre la investigación, los cuales ayudaron a mejorar el presente trabajo.

\section{REFERENCIAS}

Álvarez-Muñoz, Patricio y Mario Pérez-Montoro. 2015 “Análisis de la producción Científica y de la visibilidad Científica de Ecuador en el contexto Andino 20002013". El profesional de la información 24 (5): 577-586.

https://doi.org/10.3145/epi.2015.sep.07

Álvarez-Muñoz, Patricio y Mario Pérez-Montoro. 2016 "Políticas Científicas públicas en Latinoamérica: El caso de Ecuador y Colombia”. El profesional de la información 25 (5): 758-766. https://doi.org/10.3145/epi.2016.sep.06

Bonilla, Claudio A., José M. Merigó y Carolina Torres-Abad. 2015 "Economics in Latin America: a bibliometric analysis”. Scientometrics 105 (2): 1239-1252. https://doi.org/10.1007/s11192-015-1747-7

Borja, González-Albo y María Bordons. 2011. "Articles vs. proccedings papers: Do they in research relevance and impact? A case study in the library and Information science field". Journal of Informetrics 5 (3): 369-381. https://doi.org/10.1016/j.joi.2011.01.011

CACES (Consejo de Aseguramiento de la Calidad de la Educación Superior). 2018. Politica de Evaluación Institucional de Universidades y Escuelas Politécnicas en el marco del Sistema de Aseguramiento de la Calidad de la Educación Superior. Quito: Consejo de Aseguramiento de la Calidad de la Educación Superior. https://www.caces.gob.ec/documents/20143/152061/POL\%C3\%8DTICA+DE+EVALUACI $\%$ C3\% $\% 3 \mathrm{~N}+$ INSTITUCIONAL+DE+UNIVERSIDADES+Y+ESCUELAS+POLIT $\%$ C $3 \% 89$ CNICAS+EN+EL+MARCO+DEL+SISTEMA+DE+ASEGURAMIENTO+DE+LA++CALIDAD+DE+LA+EDUCACI\%C3\%93N+SUPERIOR. pdf/f9bed3bd-ac9b-aedc-dd75-28291a014a33 
Castillo, José Antonio. 2018. "Memorias de congresos versus artículos científicos: análisis del caso ecuatoriano". Revista Bionatura 3 (1): 1-19. https://doi.org/10.21931/RB/2018.03.01.6

Castillo, José Antonio y Michael A. Powell. 2018. "Research Impact and International collaboration: A Study of Ecuadorian Science”. Journal of Hispanic Higher Education. https://doi.org/10.1177/1538192718779169

Castillo, José Antonio y Michael A. Powell. 2019. "Análisis de la producción científica del Ecuador e impacto de la colaboración internacional en el periodo 20062015". Revista Española de Documentación Científica 42: 225. https://doi.org/10.3989/redc.2019.1.1567

CEAACES (Consejo de Evaluación, Acreditación y Aseguramiento de la Calidad de la Educación Superior). 2015. Modelo de Evaluación Institucional de Universidades y Escuelas Politécnicas; Investigación; institucionalización y resultados de la investigación. https://dptoevaluacion.sangregorio.edu.ec/wp-content/uploads/2018/04/Modelo-de-evaluacio\%CC\%81n-institucional-2016.pdf

CEPAL (Comisión Económica para América Latina y el Caribe). 2016. Ciencia tecnología e innovación en la economía digital: La situación de América Latina y el Caribe. Segunda Reunión Conferencia de Ciencia, Innovación y Tecnologías de la Información y las Comunicaciones de la CEPAL, desarrollada el 12 y 13 de Septiembre del 2016, en San José. Costa Rica. https://repositorio.cepal.org/bitstream/handle/11362/40530/3/S1600833_es.pdf

CES (Consejo de Educación Superior). 2012. Reglamento de Carrera y Escalafón del Profesor Investigador del Sistema de Educación Superior (Codificación). http://www.ces.gob.ec/doc/gaceta_ces/reglamento/reglamento\%20de\%20carrera $\% 20 \mathrm{y} \% 20$ escalafn $\% 20$ del $\% 20$ profesor $\% 20 \mathrm{e} \% 20$ investigador $\% 20 \mathrm{del} \% 20$ sistema $\% 20 \mathrm{de} \% 20$ educacin $\% 20$ superior-codificada.pdf

Chinchilla-Rodríguez, Zaida, Ricardo Arencibia-Jorge, Félix de Moya-Anegón y Elena Corera-Álvarez. 2015. "Somes patterns of Cuban scientific publication in Scopus: the current situation and challenges". Scientometrics 103, 779-794. https://doi.org/10.1007/s11192-015-1568-8

Chinchilla-Rodríguez, Zaida, Sandra Miguel y Félix de Moya-Anegón. 2015. "What factors affect the visibility of Argentinean publications in humanities and social sciences in Scopus? Some evidence beyond the geographic realm of research". Scientometrics 102, 789-810. https://doi.org/10.1007/s11192-014-1414-4

Chinchilla-Rodríguez, Zaida, Grisel Zacca-González, Benjamín Vargas-Quesada y Félix Moya-Anegón. 2017. "Latin American Scientific output in Public Health: combined analysis using bibliometric, socioeconomic and health indicators". Scientometrics 102 (1): 609-628. https://doi.org/10.1007/s11192-014-1349-9

Crespo-Gascón, Sofía, Francisco S. Tortosa y José Guerrero-Casado. 2019. "Producción de revistas científicas en América Latina y El Caribe en Scopus, Journal Citation Reports y Latindex en el área de los recursos naturales: su relación con variables económicas, ambientales y de inversión en investigación”. Revista Española de Documentación Científica" 42 (1): e224. https://doi.org/10.3989/redc.2019.1.1533 
Donaldson, Michael R. y Steven J. Cooke. 2014. "Scientific Publications: Moving beyond Quality and Quantity toward Influence”. BioScience 64 (1): 12-13. https://doi.org/10.1093/biosci/bit007

Fleitas Triana, María Sonia, Claudia Hernández Oquendo y Sandra Guerra Castillo. 2017. "Visibilidad e Impacto de La Producción Científica de La Facultad de Ingeniería Industrial de La Cujae de Cuba (2003-2012)”. Investigación Bibliotecológica: archivonomía, bibliotecología e información (Número Especial de Bibliometría): 149-185. https://doi.org/10.22201/iibi.24488321xe.2017.nesp1.57889

Fischer, Joern, Euan G. Ritchie y Jan Hanspach. 2012. "Academia's Obsession with Quantity”. Trends in Ecology E Evolution 27 (9): 473-474.

https://doi.org/10.1016/j.tree.2012.05.010

González-Albo, Borja y María Bordons. 2011. “Articles vs. proccedings papers: Do they in research relevance and impact? A case study in the library and Information science field". Journal of Informetrics 5 (3): 369-381. https://doi.org/10.1016/j.joi.2011.01.011

Guerrero Casado, José. 2017. "Producción científica latinoamericana indexada en Scopus en el área de las ciencias agropecuarias: análisis del período 1996-2016”. Idesia 35 (4): 27-33. https://scielo.conicyt.cl/pdf/idesia/v35n4/0718-3429-idesia-35-04-00027.pdf

LOES (Ley Orgánica de Educación Superior). 2010. Registro Oficial Suplemento 298 de 12 de octubre de 2010 . https:/www.educacionsuperior.gob.ec/wp-content/uploads/downloads/2014/03/LEY_ORGANICA_DE_EDUCACION_SUPERIOR_LOES.pdf

Loyola, Rafael D., José Alexandre F. Diniz-Filho y Luis Mauricio Bini. 2012. “Obsession with Quantity: A View from the South”. Trends in Ecology E Evolution 27 (11): 585 .

https://doi.org/10.1016/j.tree.2012.07.016

Miguel, Sandra. 2011. "Revistas y Producción Científica de América Latina y El Caribe: Su Visibilidad En SciELO, RedALyC y Scopus”. Revista Interamericana de Bibliotecología 34 (2): 187-199.

http://www.redalyc.org/html/1790/179022554006/

Monge-Nájera, Julián. 2002. "Cómo Hacer Ciencia En Los Trópicos”. Revista de Biología Tropical 50 (3-4): XIX-XXVIII.

http://rbt.biologia.ucr.ac.cr/attachments/resources/Como_hacer_ciencia.pdf

Monge-Nájera, Julián. 2014. "La Invalidez Del Factor de Impacto Como Indicador Del Impacto de Las Revistas Científicas Latinoamericanas”. Revista de Biología Tropical 62 (1): 9-13.

http:/www.scielo.sa.cr/scielo.php?script=sci_arttext\&pid=S0034-77442014000200031

Salvador Oliván, José Antonio, Gonzalo Marco-Cuenca y Rosario Arquero-Avilés. 2018. "Impacto de Las Revistas Españolas de Biblioteconomía y Documentación y Repercusión de Las Autocitas En Su Índice H”. Investigación Bibliotecológica: archivonomía, bibliotecología e información 32 (77): 13-30.

https://doi.org/10.22201/iibi.24488321xe.2018.77.57852

SENESCYT (Secretaría Nacional de Educación Superior, Ciencia y Tecnología). 2012. Política pública para el fomento del talento bumano en educación superior. https://issuu.com/somosciencia/docs/name6554f4 
SENESCYT. 2013. "Cuatro universidades son el pretexto simbólico para un pacto nacional para la construcción de la sociedad del conocimiento, creatividad y de la excelencia”. Boletín de prensa no. 142.

https://www.educacionsuperior.gob.ec/la-era-del-conocimiento-arranca-con-la-creacion-de-4-universidades-emblematicas/

SENESCYT. 2015. Proyecto PROMETEO. http://prometeo.educacionsuperior.gob.ec/

SENESCYT. 2016. Politicas públicas sobre tecnología e innovación en América Latina y el Caribe: Caso Ecuador.

http://www.sela.org/media/2424347/politicas-publicas-ciencia-tecnologia-e-innovacion-en-alc-caso-senescyt_ecuador.pdf

SENESCYT. 2018. Lineamientos para establecer el nivel de las publicaciones indexadas para el proceso de categorización y recategorización de investigadores.

http://acreditacioninvestigadores.senescyt.gob.ec/static/documentos_plantillas/documentos/documento_lineamientos.pdf

Tahamtan, Iman, Askar Safipour Afshar y Khadijeh Ahamdzadeh. 2016. "Factors affecting number of citations: a comprehensive review of the literature". Scientometrics 107 (3): 1195-1225.

https://link.springer.com/article/10.1007/s11192-016-1889-2

Tejedor-Estupiñán, Joan-Miguel y Ricardo-Alonso Tejedor-Estupiñán. 2019. "Visibilidad e Impacto Científico: El Caso de Las Revistas Colombianas de Economía”. Investigación Bibliotecológica: archivonomía, bibliotecología e información 33 (78): $121-141$. http://doi.org/10.22201/iibi.24488321xe.2019.78.57915

Toscanini Segale, Mauro, Antonio Aguilar Guzmán y Roberto García Sánchez. 2016. "Diagnóstico de las políticas públicas de la Educación Superior en el Ecuador”. Revista Cubana de Educación Superior 35 (3): 161-178. http://scielo.sld.cu/pdf/rces/v35n3/rces13316.pdf

Van Hoof, Hubert B. 2015. “Ecuador's Efforts to Raise Its Research Profile: The Prometeo Program Case Study”. Journal of Hispanic Higher Education 14 (1): 56-68. https://doi.org/10.1177/1538192714543664

Wouters, Paul, Cassidy R. Sugimoto, Vincent Larivière, Marie E. McVeigh, Bernd Pulverer, Sarah de Rijcke y Ludo Waltman. 2019. "Rethinking impact factors: better ways to judge a journal”. Nature, 569(7758):621-623. https://doi.org/10.1038/d41586-019-01643-3

Zhang, Lin y Wolfgang Glänzel. 2012. "Proceeding papers in journals versus the "regular" Journal publications". Journal of Informetrics 6 (1): 88-96. https://doi.org/10.1016/j.joi.2011.06.007

Para citar este texto:

Moreira-Mieles, Liggia, Juan Carlos Morales-Intriago, Sofía Crespo-Gascón y José Guerrero-Casado. 2020. "Caracterización de la producción científica de Ecuador en el periodo 2007-2017 en Scopus”. Investigación Bibliotecológica: archivonomía, bibliotecología e información 34 (82): 141-157. http://dx.doi.org/10.22201/iibi.24488321xe.2020.82.58082 